\title{
Untersuchung der Richtwirkung der Einkopplung von ebenen Wellen in eine Leitung
}

\author{
M. Magdowski und R. Vick \\ Otto-von-Guericke-Universität, Magdeburg, Germany \\ Correspondence to: M. Magdowski (mathias.magdowski@ovgu.de)
}

Zusammenfassung. Elektrische Leitungen und Kabel stellen häufig die Haupteinfallstore für elektromagnetische Felder in die daran angeschlossenen Geräte und Systeme dar. Für die Einkopplung einer ebenen Welle kann der in eine Leitung eingekoppelte Strom unter bestimmten Voraussetzungen mit Hilfe der Leitungstheorie sehr effizient bestimmt werden. Er hängt dabei von den Abmessungen der Leitung, den Leitungsabschlüssen sowie der Amplitude, der Wellenlänge und der Einfallsrichtung der ebenen Welle ab.

In dieser Arbeit wird die Abhängigkeit der Einkopplung von der Einfallsrichtung näher untersucht. Dazu werden Richtdiagramme der Einkopplung berechnet, dargestellt und hinsichtlich der mittleren und maximalen Einkopplung über alle Einfallsrichtungen und Polarisationen ausgewertet. Die Ergebnisse werden genutzt, um die maximale Direktivität der Einkopplung in eine Leitung zu bestimmen. Fasst man die Einkopplung externer Felder in eine Leitung als einen Störfestigkeitstest auf, so kann die maximale Direktivität benutzt werden, um einen Vergleich zwischen unterschiedlichen Messumgebungen wie Absorberhallen und Modenverwirbelungskammern herzustellen.

\section{Einleitung}

Gestrahlte Störfestigkeitstest sind ein Mittel zur Überprüfung der Immunität von Baugruppen und Geräten im Rahmen der elektromagnetischen Verträglichkeit. Solche Tests werden meist in herkömmlichen Testumgebungen wie Absorberhallen durchgeführt. Dabei befindet sich der Prüfling in erster Näherung im Fernfeld einer felderzeugenden Antenne und wird zu jedem Zeitpunkt von einer einzelnen ebenen Welle bestrahlt. Durch Drehung des Prüflings wird die Richtung der maximalen Einkopplung und der geringsten Suszeptibilität gesucht.

Seit etwa einem Jahrzehnt werden Modenverwirbelungskammern als alternative Messumgebungen diskutiert. Dort wird der Prüfling durch ein statistisch homogenes und isotropes elektromagnetisches Feld innerhalb eines elektrisch großen Hohlraumresonators bestrahlt. Näherungsweise lässt sich das statistische elektromagnetische Feld durch eine Überlagerung von ebenen Wellen aus allen möglichen Einfallsrichtungen, Polarisationen und Phasenlagen beschreiben (Hill, 2009). Ein großer Vorteil von Modenverwirbelungskammern ist die Erzeugung sehr hoher Testfeldstärken mit vergleichsweise geringen Verstärkerleistungen.

Ein grundlegendes Problem bei der Nutzung alternativer Messverfahren ist die Vergleichbarkeit zu den herkömmlichen, etablierten Messverfahren. Zur exakten Umrechnung der Messergebnisse ist eine genaue Kenntnis des räumlichen Einkoppeldiagramms des Prüflings bzw. eine Information über die maximale Einkopplung (näherungsweise Realisierung in Absorberhallen) und die mittlere Einkopplung (näherungsweise Realisierung in Modenverwirbelungskammern) über alle Raumrichtungen nötig. Diese Angaben lassen sich auch als Richtwirkung oder Direktivität der Einkopplung zusammenfassen und sind für praktisch alle praxisrelevanten Prüflinge nicht verfügbar. Nur für elektrisch kleine Prüflinge kann von einem dipolartigen Verhalten und einer entsprechend kleinen Direktivität ausgegangen werden. Bei elektrisch großen Prüflingen kann die Direktivität dagegen auch stark ansteigen (Krauthäuser, 2011).

\section{Leitungsmodell}

Da elektrische Leitungen und Kabel meist die Haupteinfallstore für Felder in Geräte und Systeme darstellen, wird in dieser Arbeit die Richtwirkung der Einkopplung von ebenen elektromagnetischen Wellen in Leitungen untersucht. Als Leitung wird eine Doppelleitung im Freiraum angenommen, deren Geometrie in Abb. 1 zusammen mit einer einfallenden ebenen Welle dargestellt ist. Die gleichförmige Leitung zeigt in Richtung der $z$-Achse, beginnt bei $z=0$ endet 


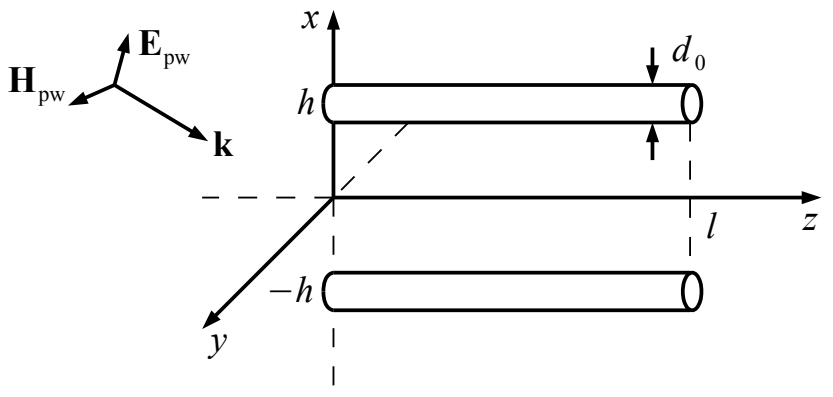

Abb. 1: Geometrie der untersuchten Doppelleitung und einer einfallenden ebenen Welle im Freiraum

bei $z=l$. Der Hinleiter befindet sich bei $x=h$, der Rückleiter bei $x=-h$. Der Durchmesser der Leiter wird mit $d_{0}$ bezeichnet. Die Leitung wird als verlustlos angenommen.

Nicht in Abb. 1 dargestellt sind die Abschlüsse der Leitung. Diese Arbeit beschränkt sich auf reelle, lineare Abschlusswiderstände, die mit $R_{1}$ am Anfang der Leitung und $R_{2}$ am Ende der Leitung bezeichnet werden.

Zur Formulierung und Lösung der Leitungsgleichungen wird die klassische Leitungstheorie (Perez, 1995) benutzt. Dabei werden folgende Vereinfachungen angenommen:

1. die Querabmaße der Leitung sind kleiner als die Wellenlänge der anregenden ebenen Wellen,

2. entlang der Leitung breiten sich nur transversal-elektromagnetische Strom- und Spannungswellen mit der Ausbreitungsgeschwindigkeit der einfallenden ebenen Wellen aus,

3. an jeder Stelle der Leitung ist die Stromsumme Null.

Besonders die letzte Vereinfachung stellt im Allgemeinen eine starke Einschränkung dar, da nur ein Gegentaktmode des Stromes erlaubt ist. Weil sich an den Enden der Leitung, an denen eine potentielle Verbindung zum Prüfling besteht, ein Gleichtaktmode bei Symmetrie der Abschlüsse aufhebt, stellt das keine große Beschränkung der Anwendbarkeit dar.

Der durch ein ebene Welle eingekoppelte Strom am Anfang und am Ende der Leitung kann besonders einfach in Form der BLT-Gleichung (Perez, 1995) formuliert werden.

$$
\begin{aligned}
{\left[\begin{array}{r}
-I(0) \\
I(l)
\end{array}\right] } & =\frac{1}{Z_{\mathrm{c}}}\left[\begin{array}{cc}
1+A_{I 1} & 0 \\
0 & 1+A_{I 2}
\end{array}\right]\left[\begin{array}{cc}
A_{I 1} & \mathrm{e}^{j k l} \\
\mathrm{e}^{j k l} & A_{I 2}
\end{array}\right]^{-1} \\
\cdot & {\left[\begin{array}{c}
-\frac{1}{2}\left(\frac{E_{z}^{\mathrm{e}}}{j\left(k-k_{z}\right)}-U_{\mathrm{t} 1}\right)\left(1-\mathrm{e}^{j\left(k-k_{z}\right) l}\right) \\
-\frac{1}{2} \mathrm{e}^{j k l}\left(\frac{E_{z}^{\mathrm{e}}}{j\left(k+k_{z}\right)}+U_{\mathrm{t} 1}\right)\left(1-\mathrm{e}^{-j\left(k+k_{z}\right) l}\right)
\end{array}\right] }
\end{aligned}
$$

Innerhalb der BLT-Gleichung werden die Reflexionsfaktoren $A_{I 1}$ und $A_{I 2}$ benutzt, die für Stromwellen wie folgt definiert sind.

$A_{I 1}=\frac{Z_{\mathrm{c}}-R_{1}}{Z_{\mathrm{c}}+R_{1}} \quad A_{I 2}=\frac{Z_{\mathrm{c}}-R_{2}}{Z_{\mathrm{c}}+R_{2}}$
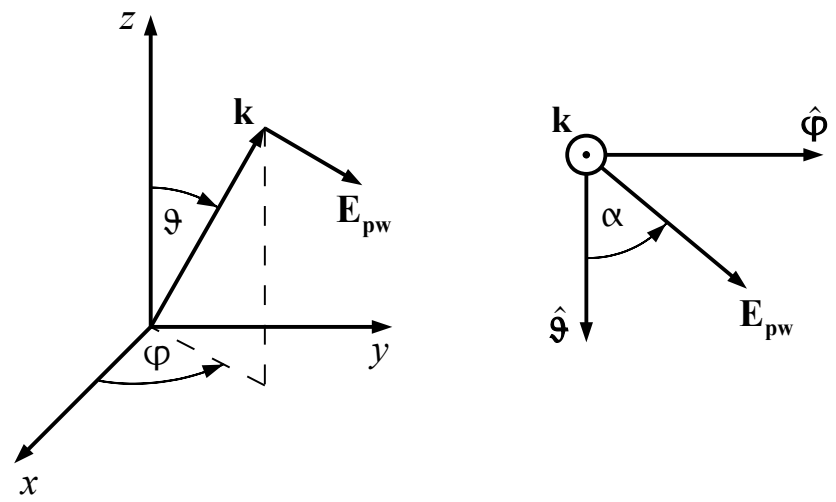

Abb. 2: Definition des Wellenvektors und der Polarisation in Kugelkoordinaten mit dem Polarwinkel $\vartheta$, dem Azimutwinkel $\varphi$ und dem Polarisationswinkel $\alpha$.

Die charakteristische Impedanz $Z_{c}$ der Leitung ergibt sich aus deren Querabmessungen.

$Z_{\mathrm{c}}=\frac{1}{\pi} \sqrt{\frac{\mu}{\varepsilon}} \cdot \operatorname{arcosh}\left(\frac{2 h}{d_{0}}\right)$

Weiterhin werden noch Ersetzungen für das einfallende elektrische Feld $E_{z}^{\mathrm{e}}$ in Richtung der Leitung und für die transversale Spannung $U_{\mathrm{t} 1}$ verwendet.

$E_{z}^{\mathrm{e}}=-j 2 E e_{z} \sin \left(k_{x} h\right) \quad U_{\mathrm{t} 1}=2 E \frac{e_{x}}{k_{x}} \sin \left(k_{x} h\right)$

Die Feldstärke der einfallenden ebenen Welle kann als komplexer Effektivwertzeiger durch folgende Gleichung beschrieben werden.

$\boldsymbol{E}_{\mathrm{pw}}(\boldsymbol{r})=E\left(e_{x} \hat{\boldsymbol{x}}+e_{y} \hat{\boldsymbol{y}}+e_{z} \hat{z}\right) \mathrm{e}^{-j(\boldsymbol{k} \cdot \boldsymbol{r})}$

Der Effektivwert der Welle wird mit $E$ bezeichnet. Die kartesischen Komponenten werden durch die Größen $e_{x}, e_{y}$ und $e_{z}$ bestimmt. Die Richtung des Wellenvektors $\boldsymbol{k}$ gibt die Ausbreitungsrichtung der Welle an. Der Betrag des Wellenvektors ist die Wellenzahl $k=\omega / c$, die sich aus der Kreisfrequenz $\omega$ und der Ausbreitungsgeschwindigkeit $c$ der Welle ergibt.

Zur einfachen Beschreibung der Einfallsrichtung der ebenen Wellen ist eine Definition in Kugelkoordinaten sinnvoll. Der Wellenvektor $\boldsymbol{k}$ und die Polarisationsrichtung der ebenen Welle sind in Abb. 2 dargestellt. Die Einfallsrichtung wird durch den Polarwinkel $\vartheta$, den Azimutwinkel $\varphi$ und den Polarisationswinkel $\alpha$ bestimmt.

Die kartesischen Komponenten des Wellenvektors ergeben sich aus den folgenden Umrechnungen.

$$
\begin{aligned}
& k_{x}=k \sin \vartheta \cos \varphi \\
& k_{y}=k \sin \vartheta \sin \varphi \\
& k_{z}=k \cos \vartheta
\end{aligned}
$$


Ebenso können die kartesischen Komponenten der elektrischen Feldstärke bestimmt werden.

$$
\begin{aligned}
& e_{x}=\cos \alpha \cos \vartheta \cos \varphi-\sin \alpha \sin \varphi \\
& e_{y}=\cos \alpha \cos \vartheta \sin \varphi+\sin \alpha \cos \varphi \\
& e_{z}=-\cos \alpha \sin \vartheta
\end{aligned}
$$

\section{Darstellung der Einkopplung als Funktion der Einfallsrichtung}

Der in eine Leitung eingekoppelte Strom ist abhängig von den Leitungsabmessungen, den Abschlusswiderständen sowie der Frequenz bzw. Wellenlänge und der Einfallsrichtung der Anregung. In Abb. 3 ist das Betragsquadrat $|I(0)|^{2}$ des Stromes am Anfang der Leitung als Funktion der Einfallsrichtung dargestellt. Das Betragsquadrat ist eine interessante Größe, da es sich analytisch einfach aus $I(0) \cdot I(0)^{*}$ bestimmen lässt und proportional zur eingekoppelten Leistung ist. Zur einfacheren Darstellung wird das Betragsquadrat mit $\left(E h / Z_{\mathrm{c}}\right)^{2}$ normiert, so dass sich eine dimensionslose Größe ergibt.

Die Abbildung zeigt acht Diagramme für Leitungslängen von $l=\lambda / 4, \lambda / 2,3 / 4 \lambda, \lambda, 5 / 4 \lambda, 3 / 2 \lambda, 7 / 4 \lambda$ und $2 \lambda$. Für jedes dieser Diagramme wird die gleiche Skalierung der Amplitudenachse verwendet. Da diese Art von Richtdiagramm nur eine zweidimensionale Darstellung erlaubt, der Strom aber von drei Winkeln abhängt, wird das Maximum über alle möglichen Polarisationswinkel $\alpha$ dargestellt. Zur Darstellung wurde der Polarwinkel entsprechend der arccos-Funktion von äquidistanten Werten zwischen -1 und 1 variiert. Diese Ungleichverteilung des Polarwinkels ist notwendig, um eine Gleichverteilung der Einfallsrichtungen über den gesamten Raumwinkel zu erreichen.

Neben dem eigentlichen Richtdiagramm der Einkopplung ist auch die Lage der Leitung durch zwei senkrechte Linien schematisch gekennzeichnet. Der Hinleiter ist die rechte der beiden Linien, der Rückleiter die linke. Der Anfang der Leitung mit $R_{1}$ als Abschluss befindet sich im Diagramm unten, das Ende mit $R_{2}$ oben. Aus der Fülle der möglichen Kombinationen von Leitungsabschlüssen wurde eine beidseitig fehlangepasste Leitung ausgewählt, da viele Leitungen nicht als hochfrequenztauglich konzipiert sind und somit in der Praxis immer mit gewissen Fehlanpassungen zu rechnen ist.

Die Abschlusswiderstände am Anfang und am Ende der Leitung wurden beispielhaft auf $R_{1}=R_{2}=3 / 2 Z_{\mathrm{c}}$ festgelegt. Durch die Fehlanpassung ergeben sich Reflexionen und Leitungsresonanzen, bei denen eine hörere Einkopplung auftritt. Diese Resonanzen treten bei $l=\lambda / 2, \lambda, 3 / 2 \lambda$ und $2 \lambda$ auf. Dort ergeben sich jeweils zwei, vier, sechs oder acht Keulen im Einkoppeldiagramm, die alle die gleiche Amplitude aufweisen. Man erkennt auch, dass das Einkoppeldiagramm bei den resonanten Leitungslängen seine Rotationssymmetrie verliert. Stattdessen tritt die maximale Ein- kopplung bei einem Azimutwinkel von $\varphi=0$ auf. Diese Einkoppelrichtung wird auch als sidefire-Anregung bezeichnet (Paul, 1994; Magdowski et al., 2010). Für die nicht resonanten Leitungslängen bzw. Frequenzen ist das Einkoppeldiagramm dagegen nahezu rotationssymmetrisch. In die kurze Leitung bzw. bei niedrigen Frequenzen wird bei einem Polarwinkel von $\vartheta=0$ ein Maximum der Einkopplung erreicht.

Obwohl die Leitung in Abb. 3 an beiden Enden mit den gleichen Widerständen abgeschlossen ist und damit auch in Richtung der Leitung symmetrisch ist, muss nicht der gleiche Strom in den Abschlusswiderstand am Anfang wie in den Widerstand am Ende eingekoppelt werden. Deshalb gibt es im Allgemeinen auch keine Symmetrie des Einkoppeldiagramms in Richtung der Leitung, da dort nur das Betragsquadrat des Stromes am Anfang der Leitung dargestellt ist. Aus der Sicht der Wellenfront jeder einfallenden Welle hat die Leitung immer ein nahes und ein fernes Ende, in die nicht zwangsweise der gleiche Strom eingekoppelt wird.

Aus Abb. 3 ist zu entnehmen, dass selbst eine gerade, gleichförmige Leitung, beschrieben durch ein einfaches Leitungsmodell, ein komplexes Einkoppeldiagramm aufweist, das je nach Frequenz und den betrachteten Leitungsabschlüssen stark variiert. Die hier betrachtete Kombination der Abschlusswiderstände an den Leitungsenden deckt dabei nur einen Bruchteil aller Möglichkeiten ab.

Eine ähnliche Untersuchung des räumlichen Einkoppeldiagramms einer Leitung wurde auch in Pissoort et al. (2012) durchgeführt. Dort wurde die Einkopplung unter Nutzung des Reziprozitätsprinzips aus dem Abstrahldiagramm einer Leitung bestimmt. Die dortigen Simulationen wurden mit Hilfe der Momentenmethode durchgeführt und erlauben auch eine Betrachtung realistischerer Leitungskonfigurationen, sind aber numerisch viel aufwendiger.

\section{Mittelwert und Maximalwert der Einkopplung}

Zur Umrechnung der Messergebnisse eines Einkoppelversuchs in eine Leitung zwischen Absorberhalle und Modenverwirbelungskammer ist nicht unbedingt eine genaue Kenntnis des räumlichen Einkoppeldiagramms der Leitung notwendig. Stattdessen ist eine Angabe über den Mittel- und Maximalwert der Einkopplung über alle Raumrichtungen und Polarisationen ausreichend. Die mittlere Einkopplung entspricht dem Erwartungswert eines Störfestigkeitstest in einer Modenverwirbelungskammer, die maximale Einkopplung wird in einer Absorberhalle erreicht.

Um den Mittel- und Maximalwert möglichst genau zu bestimmen, wurde die Anzahl der berechneten Polar-, Azimutund Polarisationswinkel gegenüber der Darstellung in Abb. 3 stark erhöht. Die Ungleichverteilung des Polarwinkels wurde aber beibehalten. Die so bestimmten Mittelwerte und Maximalwerte des Betragsquadrats des eingekoppelten Stromes am Anfang der Leitung sind in Abb. 4 als Funktion der 


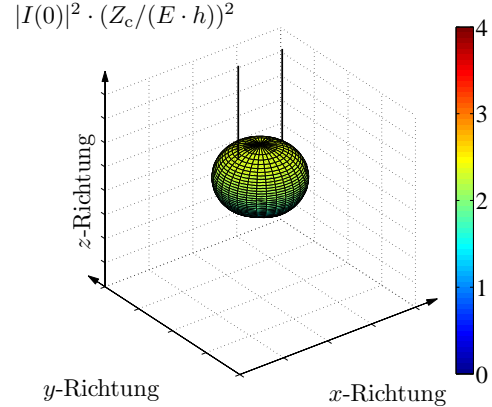

(a) für $l=\lambda / 4$

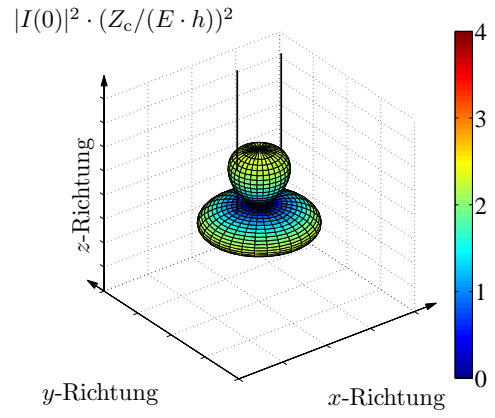

(c) für $l=3 / 4 \lambda$

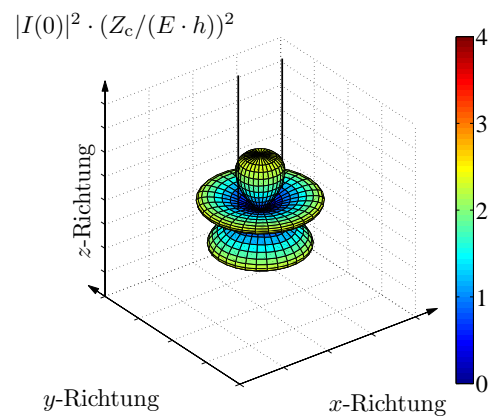

(e) für $l=5 / 4 \lambda$

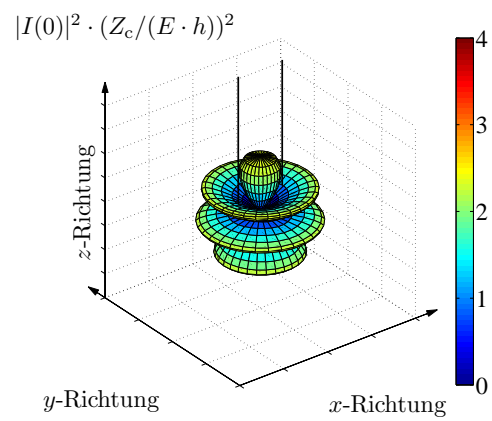

(g) für $l=7 / 4 \lambda$

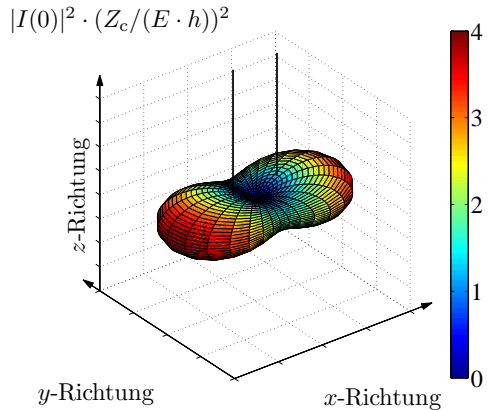

(b) für $l=\lambda / 2$

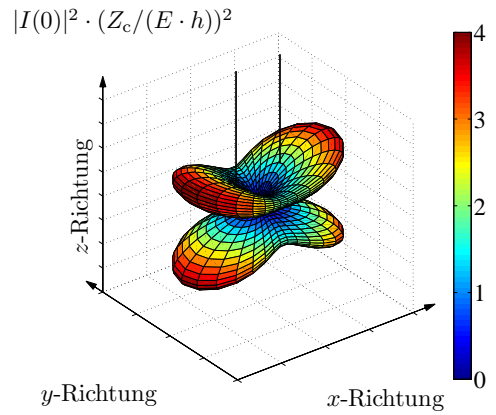

(d) für $l=\lambda$

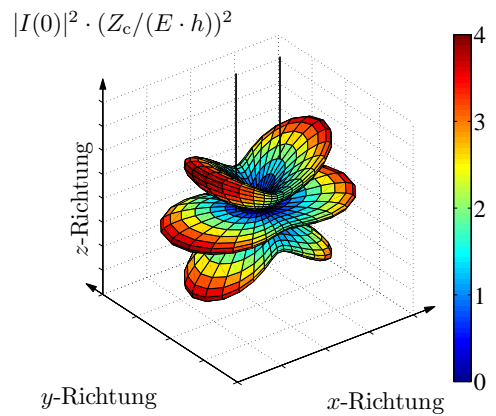

(f) für $l=3 / 2 \lambda$

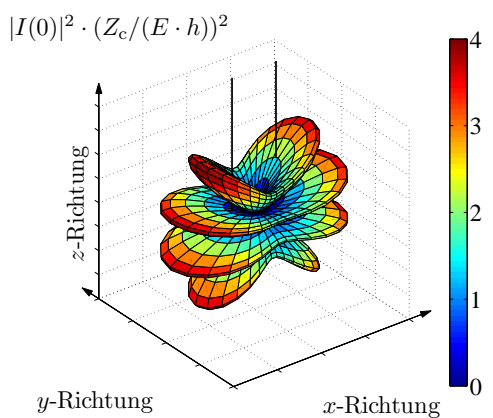

(h) für $l=2 \lambda$

Abb. 3: Betragsquadrat des eingekoppelten Stromes am Anfang der Leitung als Funktion der Einfallsrichtung der ebenen Welle für $R_{1}=R_{2}=3 / 2 Z_{\mathrm{c}}$ 


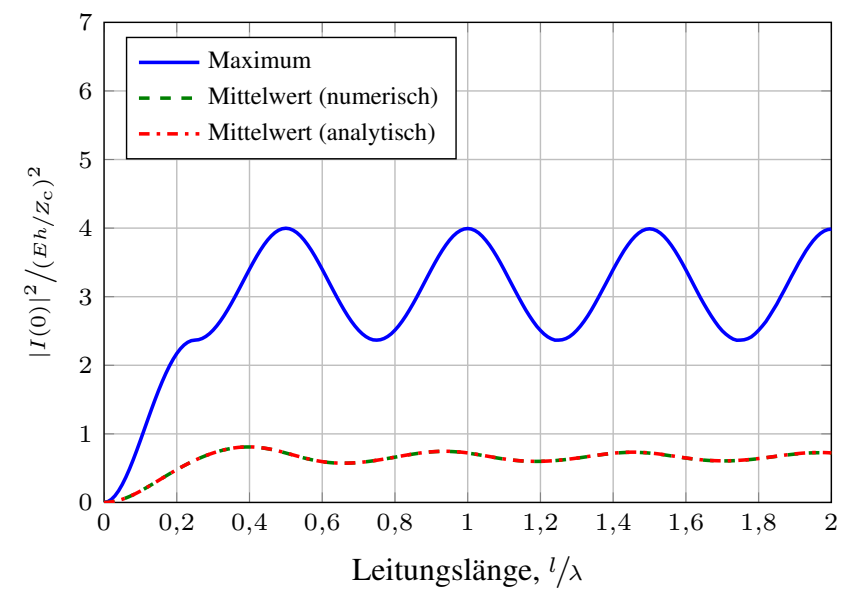

Abb. 4: Maximum und Mittelwert des Betragsquadrats des eingekoppelten Stromes am Anfang der Leitung als Funktion der Leitungslänge für $R_{1}=R_{2}=3 / 2 Z_{\mathrm{c}}$

Leitungslänge dargestellt. Zum Vergleich ist auch ein analytisch bestimmter Mittelwert der Einkopplung gezeigt, der mit Hilfe der in Magdowski and Vick (2012) angegebenen geschlossenen Gleichungen berechnet wurde. Die Leitungslänge $l$ ist auf die Wellenlänge $\lambda$ normiert. Das Betragsquadrat des Stromes ist erneut auf $\left(E h / Z_{\mathrm{c}}\right)^{2}$ bezogen, um eine dimensionslose Größe zu erhalten.

In Abb. 4 ist folgendes zu erkennen:

1. Für den Fall einer elektrisch kurzen Leitung $(l<\lambda / 4)$ steigt die mittlere und maximale Einkopplung mit wachsender Leitungslänge an.

2. Für eine elektrisch lange Leitung $(l>\lambda / 4)$ ergibt sich ein erstes Maximum der Einkopplung bei einer Leitungslänge im Bereich von $\lambda / 4<l<\lambda / 2$. Danach schwanken die maximale und mittlere Einkopplung entsprechend der Fehlanpassung der Leitung mehr oder weniger stark mit einer Periode von $\lambda / 2$. Die Amplitude der Einkopplung steigt dabei aber nicht weiter an und überschreitet auch das erste Maximum nicht wieder.

Es existiert also eine kritische Leitungslänge von $\lambda / 4<$ $l<\lambda / 2$, bei der die Einkopplung erstmals ein Höchstmaß erreicht. Eine weitere Verlängerung der Leitung wird den eingekoppelten Strom bei schmalbandiger Anregung nicht weiter erhöhen.

\section{Schlussfolgerungen für gestrahlte Störfestigkeitstests}

Der im letzten Abschnitt gezeigte Maximalwert des Betragsquadrats des eingekoppelten Stromes am Anfang der Leitung wird nun mit $\uparrow|I(0)|^{2} \uparrow$ bezeichnet. Dieser Maximalwert entspricht dem Wert der Einkopplung aus der effektivsten Einkoppelrichtung. Der ebenfalls gezeigte Mittelwert über alle möglichen Einfallsrichtungen einer ebenen Welle, der auch dem Erwartungswert bei der Einkopplung von statistischen elektromagnetischen Feldern entspricht, wird mit $\left\langle|I(0)|^{2}\right\rangle$ bezeichnet. Beide Ergebnisse können nun benutzt werden, um die Richtwirkung der Einkopplung in eine Leitung zu untersuchen und auf dieser Grundlage einen Vergleich von Störfestigkeitstests in unterschiedlichen Messumgebungen herzustellen.

Die Direktivität der Einkopplung in eine Leitung kann analog zur Direktivität der Abstrahlung eines beliebigen Strahlers als Verhältnis der Einkopplung aus einer bestimmten Richtung bezogen auf die mittlere Einkopplung aus allen Richtungen definiert werden. Zur Charakterisierung der Einkopplung wird das Betragsquadrat des eingekoppelten Stromes benutzt. Von besonderem Interesse ist die maximale $\mathrm{Di}$ rektivität, also die Direktivität in der Haupteinkoppelrichtung, in welcher der Maximalwert des Betragsquadrats des Stromes eingekoppelt wird.

$D_{\max }(0)=\frac{\uparrow|I(0)|^{2} \uparrow}{\left\langle|I(0)|^{2}\right\rangle}$

Die so bestimmte Direktivität ist in Abb. 5 als Funktion der Leitungslänge für verschiedene Kombinationen von Abschlusswiderständen dargestellt. Um eine möglichst allgemeine Aussage zu erzielen, wurden auch geringere und stärkere Fehlanpassungen als in den vorherigen Abschnitten berücksichtigt. Die dargestellten Leitungslängen sind erneut auf die Wellenlänge $\lambda$ der anregenden ebenen Wellen normiert.

Die Direktivität einer beidseitig angepassten Leitung weist die geringsten Schwankungen auf und pendelt um einen Wert von etwa Vier. Je größer die Fehlanpassung der Leitung, desto stärker werden auch die Leitungsresonanzen in der Direktivität sichtbar. Der Minimalwert von $D_{\max }(0)$ wird bei Leitungslängen von $l=\lambda / 4,3 / 4 \lambda, 5 / 4 \lambda$ usw. erreicht. In diesem Fall sind die in Abschnitt 3 gezeigten Einkoppeldiagramme auch stets rotationssymmetrisch. Besonders groß wird die Direktivität bei Leitungslängen von $l=\lambda / 2, \lambda$, $3 / 2 \lambda$ und weiteren Vielfachen von $\lambda / 2$. Bei diesen Leitungslängen verlieren die in Abschnitt 3 gezeigten Einkoppeldiagramme ihre Rotationssymmetrie und sind nur noch spiegelsymmetrisch.

Die Direktivität ist aber stets größer oder gleich Zwei und hat einen Maximalwert von etwa Acht. Die größte Schwankungsbreite der maximalen Direktivität entspricht also einem Faktor von Vier bzw. $6 \mathrm{~dB}$. Diese Schwankungsbreite würde auch der Unsicherheit bei der Umrechnung von Störfestigkeitsuntersuchungen zwischen Absorberhallen und Modenverwirbelungskammern entsprechen, wenn man hauptsächlich von einer Einkopplung über die Leitungen ausgeht und keine weiteren Informationen über den Prüfling bzw. die elektrische Länge der daran angeschlossenen Leitungen besitzt. Detaillierte Untersuchungen der Abstrahldirektivität von beliebigen Prüflingen, die zur Umrechnung von 


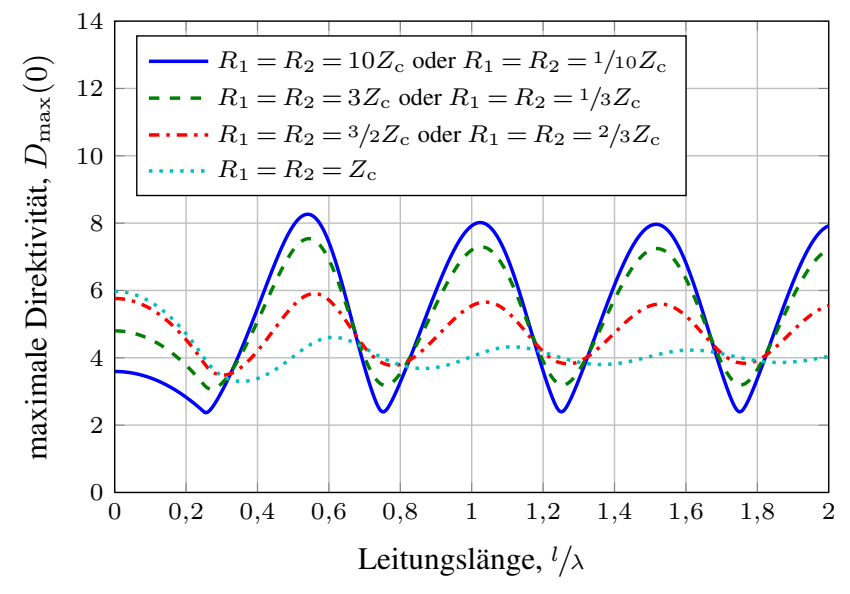

Abb. 5: Maximale Direktivität des Betragsquadrats des einkoppelten Stromes am Anfang der Leitung als Funktion der Leitungslänge für unterschiedliche Abschlusswiderstände

Störemissionsmessungen benötigt werden, sind in Krauthäuser (2011) zu finden. Dort wird aber im Allgemeinen davon ausgegangen, dass die maximale Direktivität mit der Frequenz ansteigt und für elektrisch große Prüflinge auch größer als $10 \mathrm{~dB}$ bis $15 \mathrm{~dB}$ werden kann.

Bei Störfestigkeitstests in einer Absorberhalle wird die Richtung der effektivsten Einkopplung gesucht und ein Wert von $\uparrow\left|I_{\text {Absorberhalle }}(0)\right|^{2 \uparrow}$ für den Maximalwert des Betragsquadrats des eingekoppelten Stromes erreicht. Bei Störfestigkeitstests in einer Modenverwirbelungskammer entscheidet typischerweise nicht die mittlere Einkopplung $\left\langle\left|I_{\text {Modenverwirbelungskammer }}(0)\right|^{2}\right\rangle$, sondern ebenfalls das Maximum $\left\lceil\left|I_{\text {Modenverwirbelungskammer }}(0)\right|^{2}\right\rceil$, das sich über alle vermessenen Randbedingungen ergibt. Außerdem bezieht man sich stets auf den Maximalwert einer Feldstärkekomponente. Da die statistische Verteilung einer Feldkomponente und die Verteilung des eingekoppelten Stromes gleich sind (Magdowski and Vick, 2012), ist auch das Verhältnis zwischen den entsprechenden statistischen Mittel- und Maximalwerten gleich. Somit lässt sich unter der Voraussetzung, dass das maximale Betragsquadrats einer Feldstärkekomponente in einer Absorberhalle und in einer Modenverwirbelungskammer gleich sind, folgende Formel zur Umrechnung des Betragsquadrats des eingekoppelten Stromes nutzen:

$$
\frac{\left\lceil\left|I_{\text {Modenverwirbelungskammer }}(0)\right|^{2}\right\rceil}{\uparrow\left|I_{\text {Absorberhalle }}(0)\right|^{2} \uparrow}=\frac{3}{D_{\max }(0)}
$$

Der Faktor von 3 resultiert aus der Beziehung zwischen dem Betragsquadrat der gesamten Feldstärke und einer Feldstärkekomponente in einer Modenverwirbelungskammer (Hill, 2009).

\section{Zusammenfassung}

Auf Basis der klassischen Leitungstheorie wurde das Richtdiagramm der Einkopplung ebener Wellen in eine gleichförmige und verlustlose Leitung untersucht. Aus dem Richtdiagramm wurden der Maximalwert sowie der Mittelwert des Betragsquadrats des eingekoppelten Stromes über alle Einfallsrichtungen und Polarisationen der einfallenden Wellen bestimmt. Das Verhältnis dieser beiden Größen entspricht der maximalen Richtwirkung bzw. Direktivität der Einkopplung ebener Wellen in eine Leitung. Diese Größe kann genutzt werden, um eine Korrelation von Störfestigkeitsmessungen in unterschiedlichen Messumgebungen wie Absorberhalle und Modenverwirbelungskammer herzustellen.

\section{Literatur}

Hill, D. A.: Electromagnetic Fields in Cavities: Deterministic and Statistical Theories, IEEE Press Series on Electromagnetic Wave Theory, IEEE Press, Piscataway, NJ, 1 Edn., 2009.

Krauthäuser, H. G.: Statistical Analysis of the Correlation of Emission Limits for Established and Alternative Test Sites, IEEE Transactions on Electromagnetic Compatibility, 53, 863-875, doi:10.1109/TEMC.2010.2102764, 2011.

Magdowski, M. and Vick, R.: Closed-Form Formulas for the Stochastic Electromagnetic Field Coupling to a Transmission Line With Arbitrary Loads, IEEE Transactions on Electromagnetic Compatibility, 54, 1147-1152, doi:10.1109/TEMC.2012.2193130, 2012.

Magdowski, M., Tkachenko, S. V., and Vick, R.: Visualisierung der Propagation und Reflexion von eingekoppelten Wellen auf Leitungen im Zeitbereich, in: EMV 2010 Internationale Fachmesse und Kongress für Elektromagnetische Verträglichkeit, edited by Gonschorek, K.-H., 479-486, VDE Verlag, Düsseldorf, 2010.

Paul, C. R.: Analysis of Multiconductor Transmission Lines, Wiley series in microwave and optical engineering, John Wiley \& Sons, New York, USA, 1994.

Perez, R., ed.: Handbook of Electromagnetic Compatibility, Academic Press, London, 1 Edn., 1995.

Pissoort, D., Vanhee, F., Boesman, B., Catrysse, J., Vandenbosch, G., and Gielen, G.: High-Frequency Coupling to Cables for Plane Wave and Random Wave Conditions, in: ESA Workshop on Aerospace EMC, edited by Communications, E., ESTEC, Noordwijk, Niederlande, Venedig, Italien, 2012. 